\title{
The new infrared diffuse interstellar bands in the Galactic center
}

\author{
T. R. Geballe ${ }^{1}$, F. Najarro ${ }^{2}$, D. de la Fuente ${ }^{2}$, D. F. Figer ${ }^{3}$, \\ A. J. Adamson ${ }^{1}$ and M. G. Rawlings ${ }^{4}$ \\ ${ }^{1}$ Gemini Observatory, 670 N. A'ohoku Pl., Hilo, HI 96720 USA \\ email: tgeballe@gemini.edu \\ ${ }^{2}$ Center for Astrobiology, Torrejón de Ardoz, Spain \\ ${ }^{3}$ Rochester Institute of Technology, Rochester, NY 14623, USA \\ ${ }^{4}$ ALMA-NRAO, Charlottesville, VA 22903 USA
}

Keywords. astrochemistry, ISM: abundances - lines and bands - molecules, Galaxy: center

\section{Introduction}

The diffuse interstellar bands (DIBs) are a set of absorption features, some of which are broad ("diffuse"), that are formed in the diffuse ISM. Since their discovery nearly a century ago their numbers have increased to over 500. The strongest of these are known to be ubiquitous in the universe. There is general consensus that they are produced by large carbon-bearing molecules; however, no specific identification of any single DIB has survived scrutiny. The overwhelming majority of DIBs are at optical and very near infrared wavelengths. In 1990 two DIBs were identified in $J$-band spectra, at $1.18 \mu \mathrm{m}$ and $1.31 \mu \mathrm{m}$ by Joblin et al. (1990); until recently these were the longest wavelength examples known.

\section{Discovery and general properties}

Geballe et al. (2011) recently and serendipitously discovered 13 unidentified absorption features in the $H$ band spectra of numerous hot stars in the Galactic center (GC). Suspicion that the features are DIBs was bolstered by their presence toward stars in Cygnus OB2 (Geballe

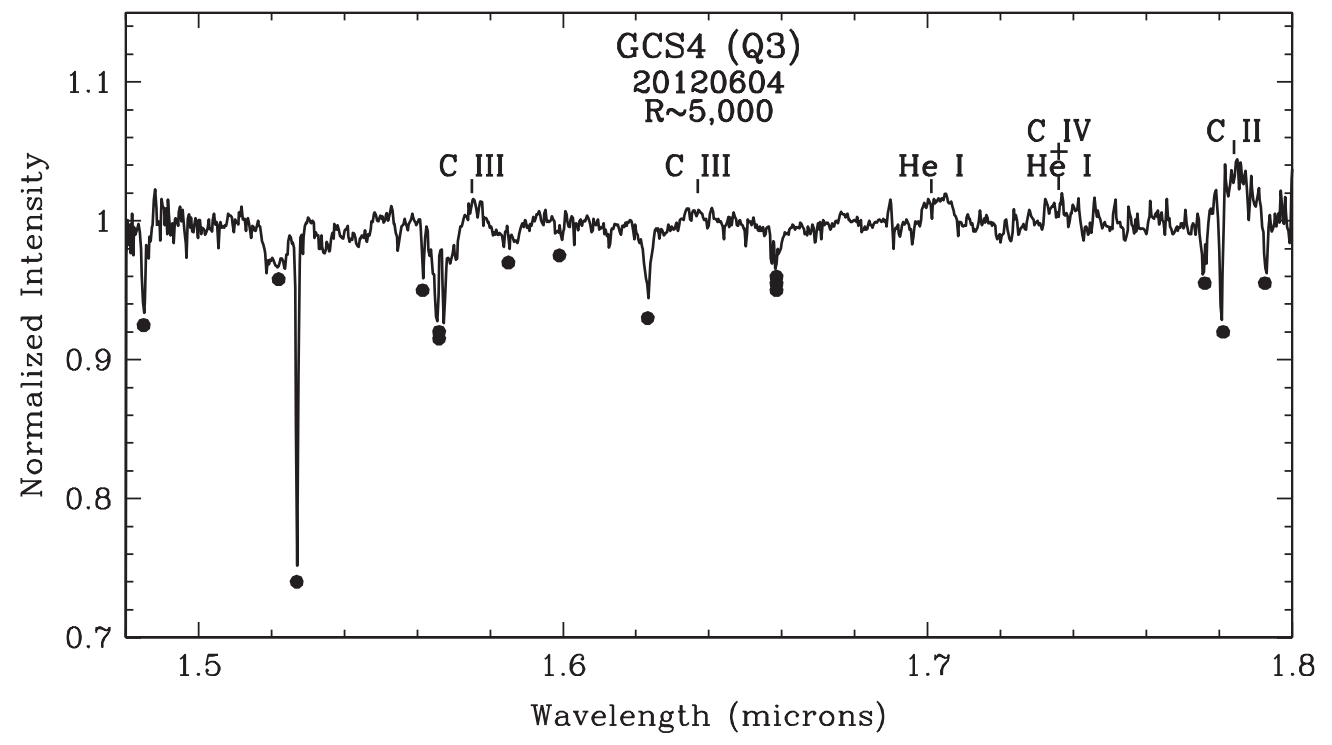

Figure 1. $H$-band spectrum of the Quintuplet source GCS4. DIBs are marked with dots. 

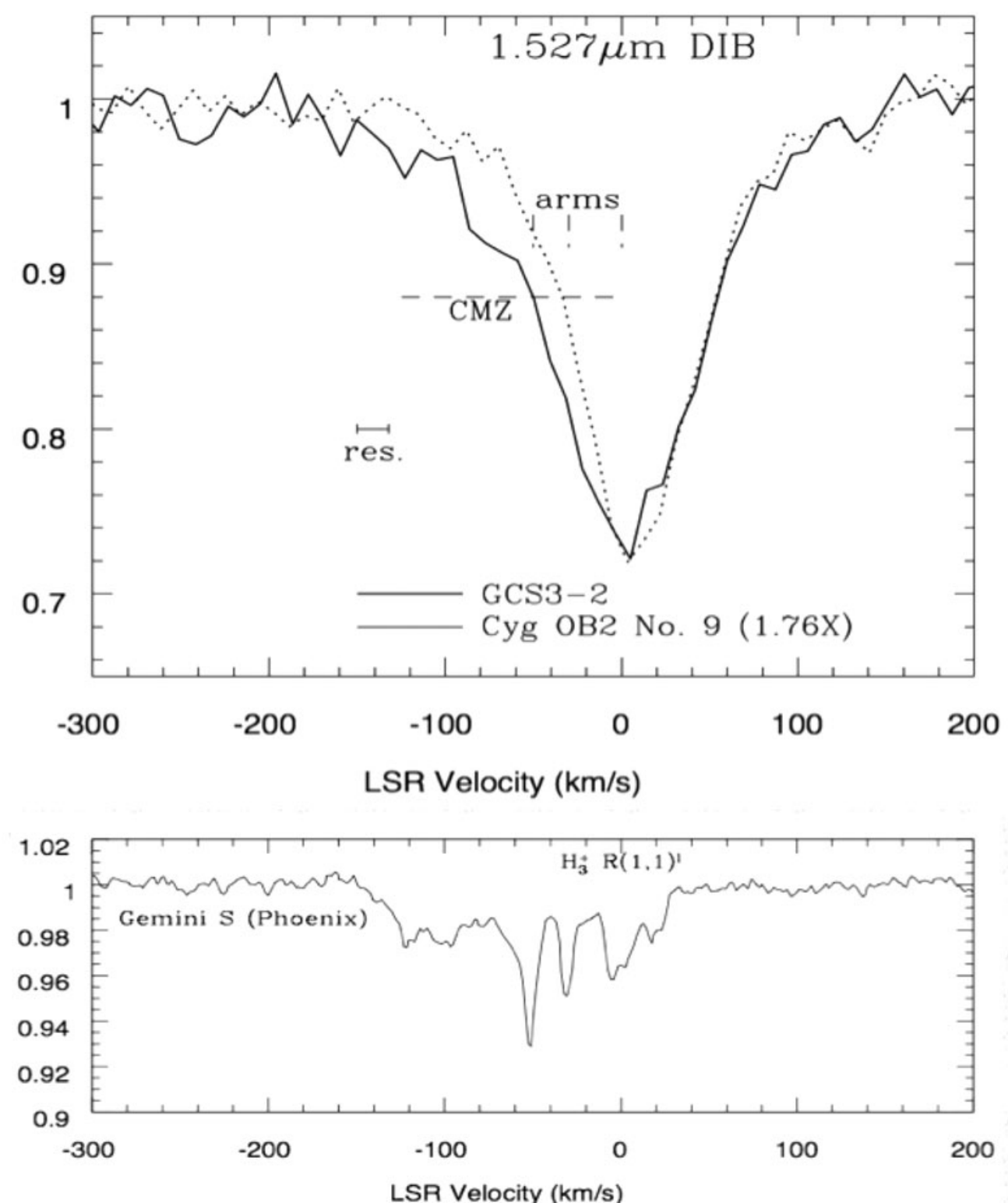

Figure 2. Comparison of $\mathrm{H}_{3}^{+}$and $1.527 \mu \mathrm{m}$ DIB profiles toward the Quintuplet source GCS3-2, with the DIB profile toward Cyg OB2 No. 9 shown as a reference.

et al. 2011), whose optical spectra contained DIBs. Whittet et al. (1997) estimate that roughly 20 magnitudes of visual extinction to the GC arise in diffuse clouds, whereas the extinction toward Cygnus OB2 is $\sim 6$ magnitudes. The DIBs in the spectra of GC stars are 3-4 times stronger than those toward stars in Cygnus OB. DIB strengths do not always scale with diffuse cloud extinction, but the above consistency increases the confidence of the identification. Altogether $\sim 15$ H-band DIBs have now been identified (Figure 1). They have a variety of profiles and strengths (Geballe et al. 2011) and are the longest wavelength DIBs discovered to date. 


\section{Distribution of the diffuse ISM toward GCS3-2}

We are attempting to use the DIBs profiles toward the GC to constrain the distribution of diffuse gas along the line of sight and the carbon abundance in the GC. Figure 2 shows the velocity profiles of the $1.527 \mu \mathrm{m}$ DIB and the $\mathrm{H}_{3}^{+} R(1,1)^{l}$ line toward the Quintuplet Cluster star GCS3-2 (Oka et al. (2005)). The major contributors to the velocity profile of this $\mathrm{H}_{3}^{+}$line are the spiral arms at $0,-30$, and $-50 \mathrm{~km} \mathrm{~s}^{-1}$ (which create the three narrow absorptions), and the central molecular zone (CMZ) which creates the broad absorption trough extending from +20 to $-140 \mathrm{~km} \mathrm{~s}^{-1}$. Comparison of this profile with those of other $\mathrm{H}_{3}^{+}$lines and infrared $\mathrm{CO}$ absorption lines show that the trough-producing gas is almost entirely diffuse whereas the gas associated with the arms must contain at least some dense clouds (Oka et al. 2005).

The profile of this DIB toward Cygnus OB2 No. 9 is also shown in Figure 2. It arises in gas with a velocity range of just a few $\mathrm{km} \mathrm{s}^{-1}$ and thus represents the intrinsic DIB shape. Comparison with the DIB profile toward GCS3-2 indicates that the majority of the extinction toward GCS32 arises in gas with velocities within $\sim 10 \mathrm{~km} \mathrm{~s}^{-1}$ of zero. Little diffuse gas is associated with the $3 \mathrm{kpc}$ and $4.5 \mathrm{kpc}\left(-30\right.$ and $\left.-50 \mathrm{~km} \mathrm{~s}^{-1}\right)$ arms on this line-of-sight, otherwise the core of the DIB profile would be broader than observed. The strength and shape of the blueshifted wing of this DIB indicates that $\sim 1 / 3$ of the extinction $\left(A_{V} \sim 6\right.$ mag) toward GCS3-2 arises in the CMZ and is associated with the warm diffuse gas producing the absorption trough in $\mathrm{H}_{3}^{+}$.

Previous estimates of the carbon abundance in the GC are 3-10 times solar (Sodroski et al. (1995). If so, then at least one of the following is correct: the DIB strength does not scale with carbon abundance; DIB carriers are depleted in the GC; the amount of extinction due to diffuse gas in the CMZ is considerably less than the above estimate. Alternatively, the carbon abundance in the GC is much closer to the solar value than the above estimate, and the DIB carriers survive the harsh conditions in the CMZ.

\section{References}

Geballe, T. R., Najaro, F., Figer, D. F., Schlegelmilch, B. W., \& de la Fuente, D. 2011, Nature 346,729

Joblin, C., Maillard, J. P., d'Hendecourt, L., \& Leger, A. 1990, Nature 346, 729

Oka, T., Geballe, T. R., Goto, M., Usuda, T., \& McCall, B. J. 2005, ApJ 632, 882

Sodroski, T. J., et al. 1995, ApJ 452, 262

Whittet, D. C. B., et al. 1997, ApJ 490, 729 